Review

\title{
Applications of Optical Interferometer Techniques for Precision Measurements of Changes in Temperature, Growth and Refractive Index of Materials
}

\author{
Rami Reddy Bommareddi \\ Department of Physics, Alabama A\&M University, P.O. Box 1268, Normal, AL 35762, USA; \\ E-Mail: rami.bommareddi@aamu.edu; Tel.: +1-256-372-8101; Fax: +1-256-372-5622
}

Received: 13 December 2013; in revised form: 16 March 2014 / Accepted: 27 March 2014 /

Published: 5 May 2014

\begin{abstract}
Optical metrology techniques used to measure changes in thickness; temperature and refractive index are surveyed. Optical heterodyne detection principle and its applications for precision measurements of changes in thickness and temperature are discussed. Theoretical formulations are developed to estimate crystal growth rate, surface roughness and laser cooling/heating of solids. Applications of Michelson and Mach-Zehnder interferometers to measure temperature changes in laser heating of solids are described. A Mach-Zehnder interferometer is used to measure refractive index and concentration variations of solutions in crystal growth experiments. Additionally, fluorescence lifetime sensing and fluorescence ratio method are described for temperature measurement. For all the above techniques, uncertainty calculations are included.
\end{abstract}

Keywords: Optical heterodyne detection; Michelson interferometer; Mach-Zehnder interferometer; laser cooling of solids; laser heating of solids; fluorescence lifetime sensing; fluorescence ratio method; optical sensing of temperature; Interferometric measurement of crystal growth

\section{Introduction}

Optical testing/assessment of optical surfaces are usually done using interferometers. So, a series of interferometers were designed based on the division of amplitude or wavefront [1]. Interference occurs whenever two coherent beams are superimposed; one of them is reflected by a reference surface and the other by a test surface. Newton demonstrated concentric fringes in an air gap between a convex surface and a flat surface. Newton's rings are easily disturbed by vibrations and so this technique is not 
used for routine testing of optical surfaces. In a Fizeau interferometer, the reference and test flats are separated by an air gap. Light reflected from these two flats generate interference fringes. Young demonstrated interference fringes by the division of wave fronts using a double slit experiment. Now-a-days, similar setups are used to measure coherent properties of light sources. Albert Michelson configured an interferometer which bears his name, to measure the speed of light. Later on, similar interferometer configurations were used for a variety of experiments. Twyman-Green (T-G) interferometer uses a collimated beam which is considered a modified version of the Michelson interferometer. Mach-Zehnder (M-Z) interferometer could be modified to a variety of experimental situations. In general, T-G, M-Z, Fizeau and shearing interferometers facilitate the testing of large optical surfaces. Most of the instruments are two-beam interferometers whereas Fabry-Pérot (F-P) interferometer is based on multiple beam interference. In the pre laser era, interferometers utilized either a sodium lamp or a mercury lamp as light source. After the advent of lasers, majority of the interferometers are equipped with HeNe lasers, because they produce stable laser sources whose beam parameters are superb. F-P etalons and interferometers are utilized extensively in laser design and to monitor single mode laser beams. Michelson type interferometers are also used in the design of wave meters [1]. Homodyne interferometer uses a single wavelength laser whereas a heterodyne interferometer uses a two frequency laser. Both of them are used in metrology instruments. Sagnac developed a common path interferometer in which the two beams travel in counter propagating directions [1]. Sagnac interferometer is very stable and hence it is used in sensing rotations. Now-a-days, interferometers can be setup using free space optics, optical waveguides or fiber optics. Fringe analysis and phase retrieval became easy and faster with the use of digital computers. In particular, phase shifting and dynamic interferometer techniques are becoming popular.

In situ monitoring of growth rate and temperature is crucial in vapor deposition, molecular beam epitaxy (MBE) and crystal growth [2]. Surface micro-irregularities limit storage capacity of magnetic media. Surface roughness also limits the resolution of images and the resolving power of optical elements. There are several ex situ techniques based on scanning electron microscope (SEM), transverse electron microscope (TEM), X-Ray diffraction (XRD) and atomic force microscope (AFM), for assessment or characterization. However, for in situ measurements, non-contact and non-destructive techniques are preferred.

Temperature is an important parameter in many industrial environments [3]. The development of temperature sensors has been continually evolving for more than two centuries. Any material whose property quickly changes proportionate to temperature can be used as a thermometer. This property can be pressure, volume, length, resistance, refractive index, color, etc. Resistive thermometers are susceptible to electromagnetic interference; thermocouples are not suitable in corrosive atmospheres, and liquid thermometers are not suitable for high temperature measurements. Harsh environments such as combustion or jet plume studies require reliable temperature sensors [3]. In optically pumped lasers, thermal effects limit the performance of laser materials and the beam quality [4]. The temperature of an optical material may change along the path of the laser beam. For such cases, surface mounted thermocouples do not reveal the accurate material temperature along the beam path, because a temperature gradient exists. So, alternate techniques are required for many such cases for the measurement of either large or small temperature changes. Whenever conventional techniques are not suitable, optical metrology techniques are being developed. Optical sensors have gained importance 
after laser sources are incorporated into metrology instruments. In this article, optical techniques are reviewed to measure growth rate and temperature changes. High precession measurements involve changes in phase measurement [5]. Applications of Michelson, Mach-Zehnder and optical heterodyne interferometers are described in detail, for in situ measurement of material temperature changes, crystal growth rate measurement, and refractive index and concentration changes of solutions. Fluorescence intensity and lifetime based techniques are also reviewed for temperature measurements. These techniques are useful in harsh environments and also in other situations, where conventional methods are not applicable.

\subsection{Survey Optical Techniques for Growth Rate Measurements}

Interferometry has applications in science and industry including precision surface topography, film growth monitoring, temperature sensing, and concentration mapping [5]. In general, interferometry can be used to measure films, which are thicker than one fourth of laser wavelength, whereas ellipsometry can be used for thin film characterization. In ellipsometry, the polarization of the incident light is rotated after reflection from the sample, which is detected by an analyzer [6]. Phase shifting interferometry (PSI) [7], vertical scanning interferometry (VSI), Moiré interferometry [8] and holographic interferometry [9] techniques are computer based techniques and their data processing times are faster [7]. In PSI, the relative phase of reference beam is varied in a continuous manner by applying discrete voltages to a PZT mounted mirror, and phase shifted interferograms are recorded sequentially. The interferograms are recorded with a CCD camera, and a computer measures the intensity variation for various phase shifts to characterize the object [7]. Measurement sensitivity of PSI is higher and small changes in concentration, temperature and density can be determined. VSI uses white light and is useful to map rough surfaces but its sensitivity is less than that of PSI [10]. Laser reflectance interferometry (LRI) involves measuring the intensity of a laser beam reflected from the front and back surface of a material/film under growth [11]. Film thickness and growth rate can be measured using LRI. However, surface roughness and bulk absorption/reflectance, adversely affect the use of this technique after some material growth, as it decreases the reflectivity, and hence the oscillation amplitude. Michelson and Mach-Zehnder interferometers are used in real time for growth rate measurements and concentration field measurements onboard the international space station [12]. Differential laser interferometer technique was used to measure thermal expansion of solids, by using a stepped sample. Light is reflected from both the surfaces. Two fringe signals that are $90^{\circ}$ out of phase are monitored simultaneously [13]. Film thickness was measured by a modified Michelson interferometer in which one mirror was replaced by a step. A double interference pattern was obtained which depicted a shift between the patterns. The patterns were obtained from the substrates with and without the film. Film thickness was calculated from the shift.

\subsection{Survey of Optical Sensor Technologies for Temperature Measurement}

Optical measurements involve the detection of light. Any parameter that changes with temperature, for example, changes in emission intensity, lifetime, optical path length or phase of an electromagnetic wave can be used to estimate the sample temperature. Raman scattering is the principle behind the operation of distributed temperature sensing (DTS) device [14]. If a fiber's temperature changes at one 
or more points along the length of a fiber, thermal effects induce lattice vibrations locally, and a propagating pulsed laser beam undergoes Raman scattering at those points. The ratio of anti-Stokes to Stokes line intensities is used to derive the fiber temperature. Commercial devices measure temperatures up to $700{ }^{\circ} \mathrm{C}$ with a linear resolution of $1 \mathrm{~m}$. Fiber Bragg grating temperature sensors are used within nuclear power facilities to monitor reactor core temperature and avoid the possibility of nuclear meltdowns [15]. After the advent of ultraviolet LEDs, frequency domain lifetime sensing [16] was employed for temperature measurement. A modulated light (MHz-GHz) source excites luminescence from the sample. The resulting luminescence is also modulated, and shifted in phase, from that of the exciting light source. The detector output is demodulated and the lifetime is derived from the measured phase shift. In differential luminescence measurement (DLM), changes in luminescence spectrum of a sample are monitored as a function of temperature. Spectral subtraction reveals whether the material is heating or cooling [17]. This technique is fast and achieves sub millikelvin $(\mathrm{mK})$ resolution. Another high temperature probe design involves an optical fiber made of $\mathrm{Y}_{2} \mathrm{O}_{3}$, or another [18]. One end of the fiber is coated with rare-earth ion doped film that is in contact with the surface under measurement. The light output of the fiber passes through a band pass filter (or a spectrometer) before being detected, whose intensity is related to the sample temperature. This device is useful for temperature measurement from $400-2000{ }^{\circ} \mathrm{C}$, and operates even in reactive and corrosive environments. It has a fast response time of $1 \mathrm{~ms}$ and a temperature resolution of $1{ }^{\circ} \mathrm{C}$. In spite of all the developments, there is no universal device that is suitable for all environments. So, optical metrology is continually evolving with different applications. Any technique that involves phase measurement is more accurate than fringe counting techniques. Here, we will review the theory and the applications of interferometers and fluorescence techniques for scientific research and industrial measurements.

\section{Experimental Details}

\subsection{Optical Heterodyne Technique}

In general, a typical interferometer involves two beams of which one is a reference beam and the other is a measurement beam. Measurement beam either gets reflected or passes through the material under investigation. Depending on the application, these experiments can be setup in several different configurations. We will review some unique applications of optical heterodyne interferometer in this section. Interferometry deals with either fringe counting or phase measurement $[4,5]$. In a fringe counting experiment, an ordinary $\mathrm{HeNe}$ or another low power laser of long coherence length is required. For phase measurements, a stabilized two frequency Zeeman laser is required [19]. If a Zeeman laser is not available, the required two frequencies can be produced with modulators. Figures 1 and 2 depict Michelson and Mach-Zehnder interferometer configurations, required for the implementation of optical heterodyne interferometer technique. A milliwatt Zeeman laser produces two collinear and orthogonally polarized beams, whose frequencies differ by $1.5 \mathrm{MHz}$ for a HP laser and $250 \mathrm{kHz}$ for an OPTRALITE laser. These frequencies are spatially separated by a polarizing beam splitter (PBS) and one beam gets retro-reflected by a fixed mirror, hereafter called the reference beam, and the other beam is retro-reflected by a film/crystal under growth, hereafter called the measurement 
beam (Figure 1). The reflected beams are recombined by the PBS and then directed to a detector (Figure 1). This technique was implemented for the measurement of LAP crystal growth from solution [20] and the surface roughness of coated mirrors [21] in real time. For some applications, a setup similar to that of a Mach-Zehnder interferometer is preferred. The experimental configuration of optical heterodyne detection technique needed for the investigation of laser cooling and heating of solids is described in Figure 2. The $\mathrm{p}(\mid)$ and $\mathrm{s}(\bullet)$ polarized beams are spatially separated by a PBS. For the measurement of crystal growth or film deposition rate, a Michelson interferometer configuration is convenient (Figure 1). For the measurement of laser cooling or heating of solids, a Mach-Zehnder interferometer configuration is ideal (Figure 2). Based on the optical configuration, the mathematical model has to be developed accordingly.

Figure 1. Optical configuration of the experimental setup for growth rate measurement $\mathrm{p}(\mid)$ and $\mathrm{s}(\bullet)$-polarization components are separated by a PBS [20].

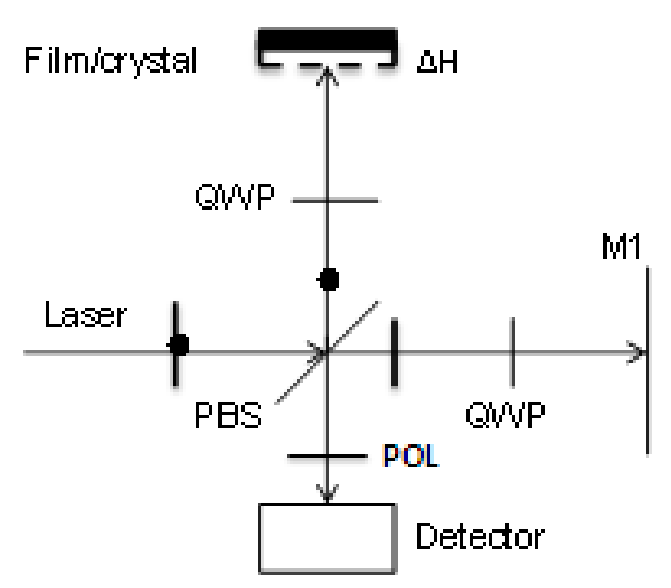

Figure 2. Mach-Zehnder interferometer configuration; p $(\mid)$ and $s(\bullet)$-polarization components are spatially separated by a PBS.

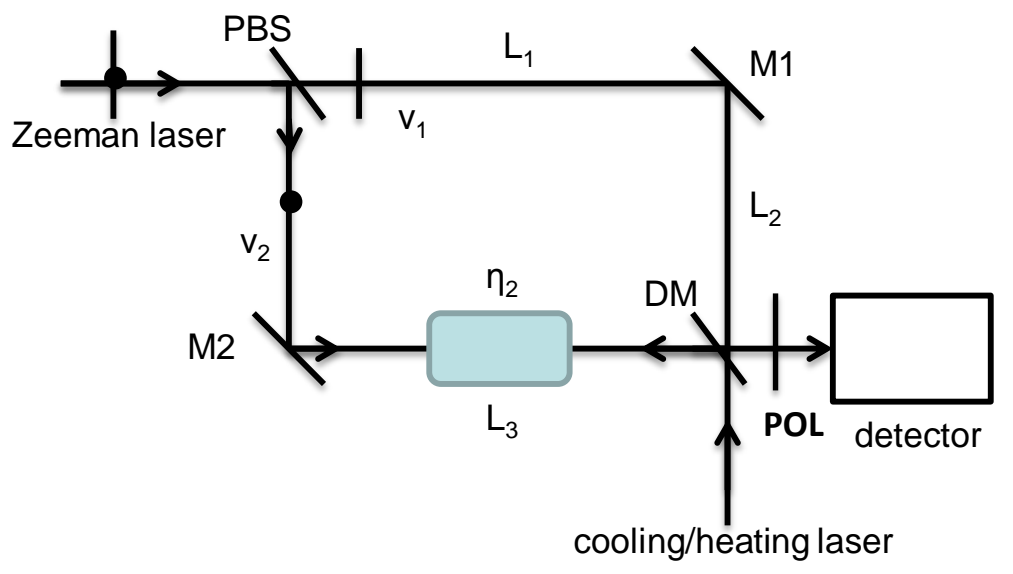




\subsection{Rare-Earth Luminescence Measurements}

Rare-earth luminescence is used to estimate sample temperature. For this purpose the system has to be calibrated. So, luminescence spectra and lifetimes were measured as a function of sample temperature. A sample compartment was machined at one end of an iron rod of $0.0254 \mathrm{~m}$ diameter, and $0.3 \mathrm{~m}$ in length. The rod was inserted into a tube furnace except for the sample chamber that remains outside (Figure 3). The sample is in contact with the metal rod on five sides. The top surface of the sample is exposed to air [22]. The laser beam enters the sample chamber from the side, through a millimeter size hole, and exits from the opposite side (see the inset, in Figure 3). A fiber bundle collects fluorescence from the top of the sample, whose other end is attached to a medium resolution spectrometer. A block diagram of the setup is shown in Figure 4. The use of a spectrometer minimizes the blackbody radiation entering the detector. A photomultiplier tube (PMT) is mounted on the exit slit for the detection of light. PMT output is acquired by a computer for spectral recording. A mechanical chopper was used to pulse the continuous wave laser beams. The amplified output of the PMT was acquired by a multichannel scaler for lifetime measurement. Single crystals were obtained from Optovac, Inc., and Scientific Materials Corp, and glasses were made in our laboratory. The melting points of YAG and $\mathrm{LaF}_{3}$ crystals are respectively 1940 and $1493{ }^{\circ} \mathrm{C}$ and the latter have small phonon frequency. These materials are used in lifetime sensing as well as interferometer methods for temperature measurement.

Figure 3. Photograph of the sample heating chamber in fluorescence lifetime sensing setup. Inset shows the sample compartment [22].

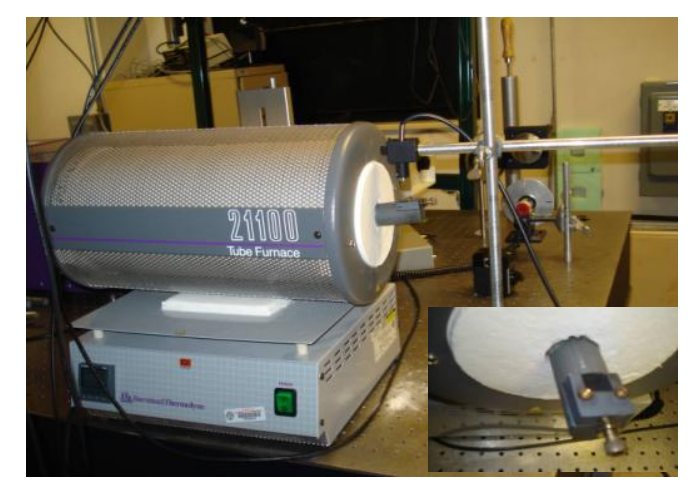

Figure 4. Block diagram of the fluorescence lifetime sensing setup [22].

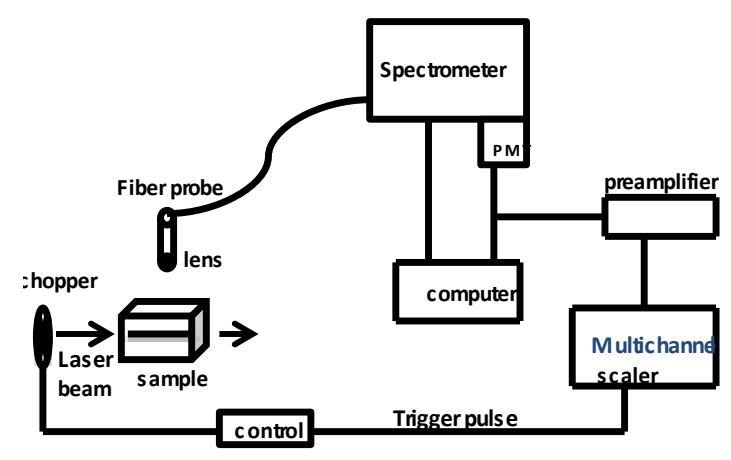




\section{Principle of the Optical Heterodyne Detection Technique}

Optical frequencies are of the order of $6 \times 10^{14} \mathrm{~Hz}$ or larger. When such a wave falls on a detector, it does not respond to such fast variations. So, the detector output is a DC current whose amplitude is proportional to the intensity of light (Figure 5). The two Zeeman laser frequencies $v_{1}$ and $v_{2}$ are slightly different, and their difference $\Delta v=v_{2}-v_{1}$, is in the radiofrequency (rf) range. When two such optical waves, $v_{1}$ and $v_{2}$, fall on a detector, its output is a DC current that is modulated by the rf current at $\Delta v$. The frequency of the rf component equals the difference between the two optical frequencies $(1.5 \mathrm{MHz}$ for HP 5501A laser, and $250 \mathrm{kHz}$ for OPTRALITE laser). If the two beams travel slightly different path lengths and then fall on a detector, the resultant amplitude is given by

$$
\mathrm{E}=\mathrm{E}_{1}+\mathrm{E}_{2}=\mathrm{A} \exp \mathrm{i}\left(\mathrm{k}_{1} \mathrm{z}_{1}-\omega_{1} \mathrm{t}\right)+\mathrm{A} \exp \mathrm{i}\left(\mathrm{k}_{2} \mathrm{z}_{2}-\omega_{2} \mathrm{t}\right)
$$

where $\mathrm{A}$ is the amplitude, and $\omega_{\mathrm{i}}, \mathrm{k}_{\mathrm{i}}$ and $\mathrm{z}_{\mathrm{i}}(\mathrm{i}=1,2)$ are the angular frequency, wavenumber and path length of each wave. Detector output current, I is proportional to the intensity of the beams,

$$
\mathrm{I}=\mathrm{EE}^{*}=\mathrm{I}_{0}+\mathrm{I}_{\mathrm{rf}}
$$

where

$$
\mathrm{I}_{\mathrm{rf}}=\mathrm{I}_{0} \cos (2 \pi \Delta v \mathrm{t}+\Delta \Theta(\mathrm{z}))
$$

where $\Delta \Theta(z)=\Theta_{1}\left(z_{1}\right)-\Theta_{2}\left(z_{2}\right) . \Theta_{1}\left(z_{1}\right)$ is the phase of the reference beam and $\Theta_{2}\left(z_{2}\right)$ is the phase of the measurement beam. As the material grows, optical path length travelled by the measurement beam decreases, and hence its phase, $\Theta_{2}\left(z_{2}\right)$ changes. Consequently, the phase difference between the two waves, $\Delta \Theta(\mathrm{z})$ changes continuously as the material grows. $\Delta \Theta(\mathrm{z})$ is related to the path length change as

$$
\Delta \Theta(\mathrm{z})=(2 \pi / \lambda) \Delta \mathrm{z}
$$

where $\lambda$ is the wavelength corresponding to the center frequency of the Zeeman laser, the roundtrip change in path length, $\Delta \mathrm{z}=2 \Delta \mathrm{H}$ and $\Delta \mathrm{H}$ is the change in thickness.

$$
\Delta H=(\lambda / 4 \pi) \Delta \Theta
$$

Figure 5. Conceptual diagram of optical heterodyne detection. Rectangle represents the detector.
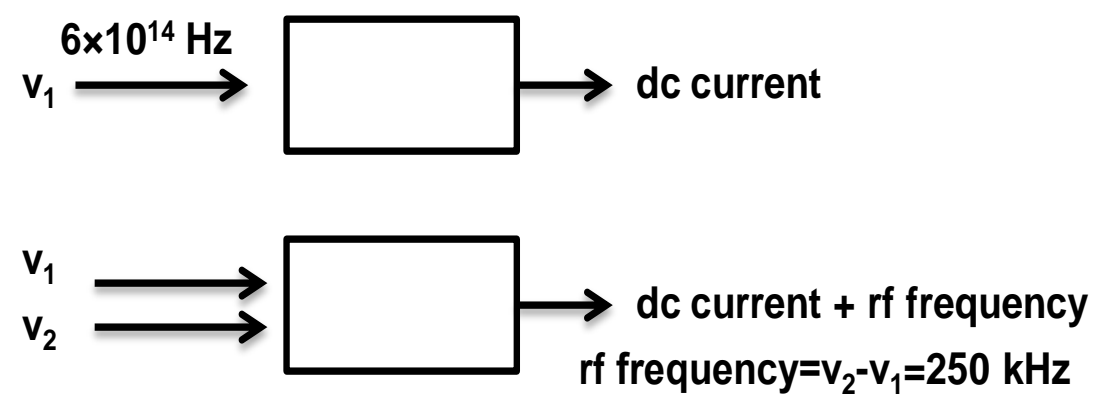

$\Delta \Theta$ is measured by the experiment. The wavelength has to be divided by the refractive index of the medium if the sample is in a solution whose refractive index is more than 1. OPTRALITE laser head 
provides the $250 \mathrm{kHz}$ rf frequency for reference purposes. For a HP laser the reference beam has to be derived from a detector output. The reference and measurement rf frequencies (detector outputs) are given to the two inputs of a HP 3575A phase meter, whose output (in DC volts) is proportional to the phase difference between the two rf inputs.

\subsection{Application of Optical Heterodyne Detection Technique for in situ Measurement of Crystal Growth}

We applied this technique for crystal growth rate measurement [20]. The experimental configuration is similar to that shown in Figure 1. The phase meter output is shown in Figure 6. The phase meter output steadily increases between $-\pi$ and $+\pi$, and immediately resets, as per the instrument design. The smallest value of $\Delta \mathrm{H}$ that can be measured is limited by the resolution of the phase measurement device employed, which equals $\Delta \Theta$. The resolution of HP $3575 \mathrm{~A}$ phase meter is $0.1^{\circ}$. For $\lambda=632.8 \mathrm{~nm}$, and $\Delta \Theta=0.1^{\circ}$ (equal to phase resolution), thickness (or height) changes can be measured to an accuracy of $0.1 \mathrm{~nm}$. For a better understanding, the data of Figure 6 are plotted in Figure $7 \mathrm{a}$ as phase change versus time. The slope reveals a phase change of $9.5 \%$ which corresponds to a growth rate of $6.1 \mathrm{~nm} / \mathrm{s}$. If the experimental setup is located in a noisy environment, it will impact the measurement sensitivity [20]. Background noises such as thermal drift, mechanical vibration may adversely affect the measurement. To address this problem, crystal growth chamber was replaced by a mirror and the detector output was monitored for an hour. The resulting noisy signals are shown in Figure $7 \mathrm{~b}$. The root-mean-square drift in phase change was $1.3^{\circ}$ which corresponds to a path length change of $0.8 \mathrm{~nm}$ in LAP solution, since the solution refractive index is 1.3565 . This technique works equally well for thin film growth rate measurement. The only requirement is that the crystal/film surface has to reflect the laser light.

Figure 6. In situ measurement of crystal growth rate by the heterodyne detection technique [20].

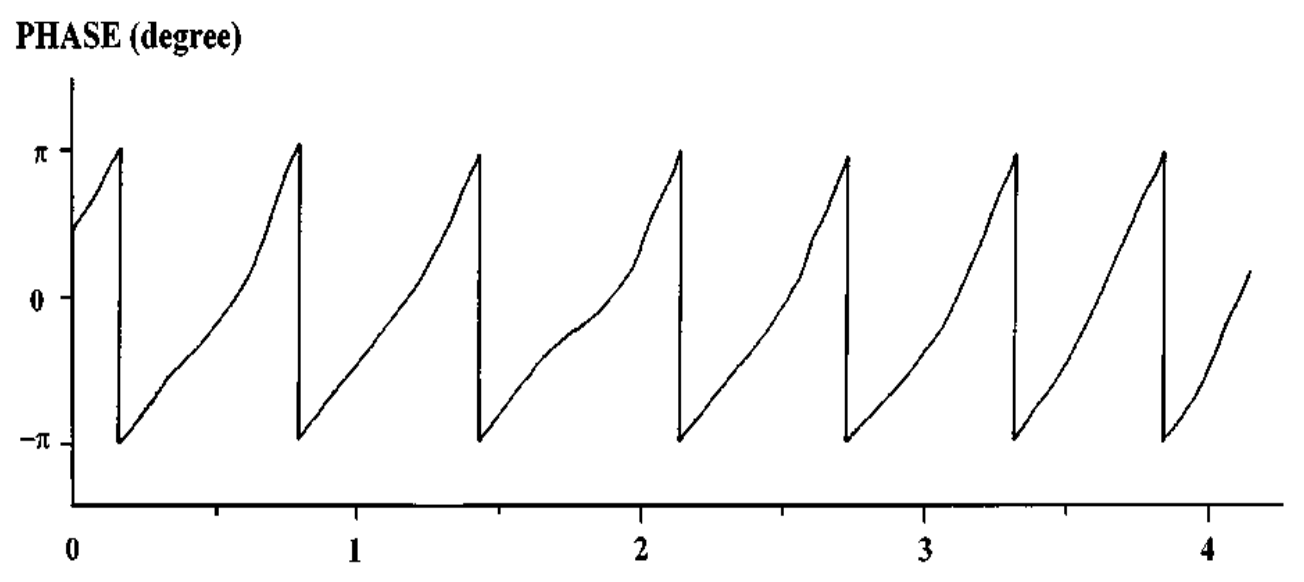

TIME (min.) 
Figure 7. (a) A plot of heterodyne signal-phase versus time in a crystal growth experiment [20]; (b) Stability measurement of heterodyne detection in the Michelson interferometer setup [20].

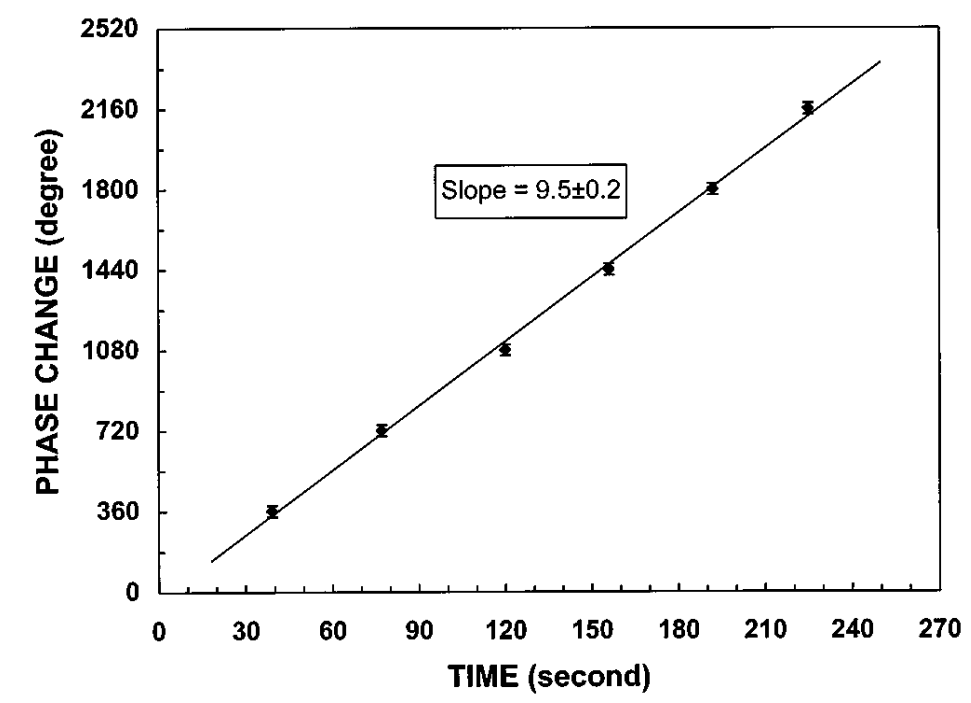

(a)

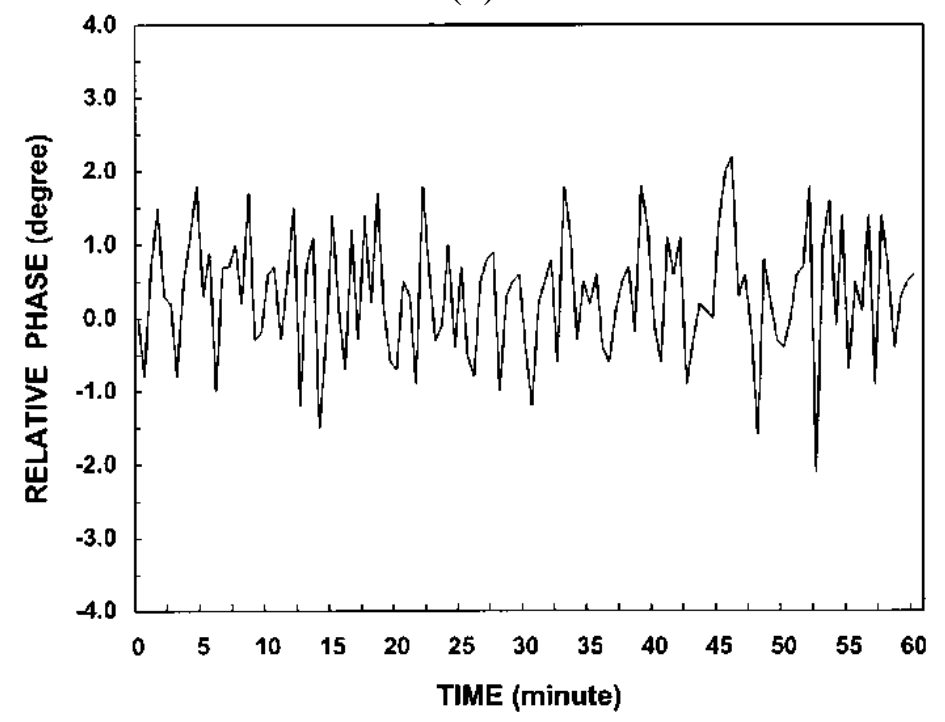

(b)

\subsection{Application of Optical Heterodyne Detection Technique for Surface Roughness Measurement}

The experimental configuration for the measurement of surface roughness of coated mirrors is different from that of crystal growth rate measurement. For this experiment, a HP Zeeman laser was used. Reference rf wave was generated by mixing the optical waves (10\% of the laser power) in a detector. Measurement rf wave was generated by launching the beams to an air-lifted mirror that can be rotated (Figure 8). The p- and s- polarization components are spatially separated by a Wollaston prism and focused at two different points on the air-lifted mirror (inset of Figure 8). One of the spots is incident at a point that is coincident with the axis of rotation. The other polarization component is slightly displaced from the axis of rotation. The positions of the beams are fixed in space. However, when the mirror is rotated, the off axis beam encounters different points in a circle. If the surface is 
rough, the path length travelled by the off axis beam (measurement beam) changes. As a result, the phase of the rf signals (detector output) changes continuously. Consequently, the phase meter output changes at each step, proportionate to the phase difference between the reference and measurement waves (rf). The phase meter output is acquired by a computer for further processing. This technique was highly sensitive, so it revealed $0.1 \mathrm{~nm}$ level surface roughness of coated mirrors [19]. However, in our laboratory we were able to measure roughness of $1 \mathrm{~nm}[21]$.

Figure 8. A block diagram of the optical heterodyne detection setup for surface roughness measurement. Inset shows the beam configuration on the mirror surface [21].

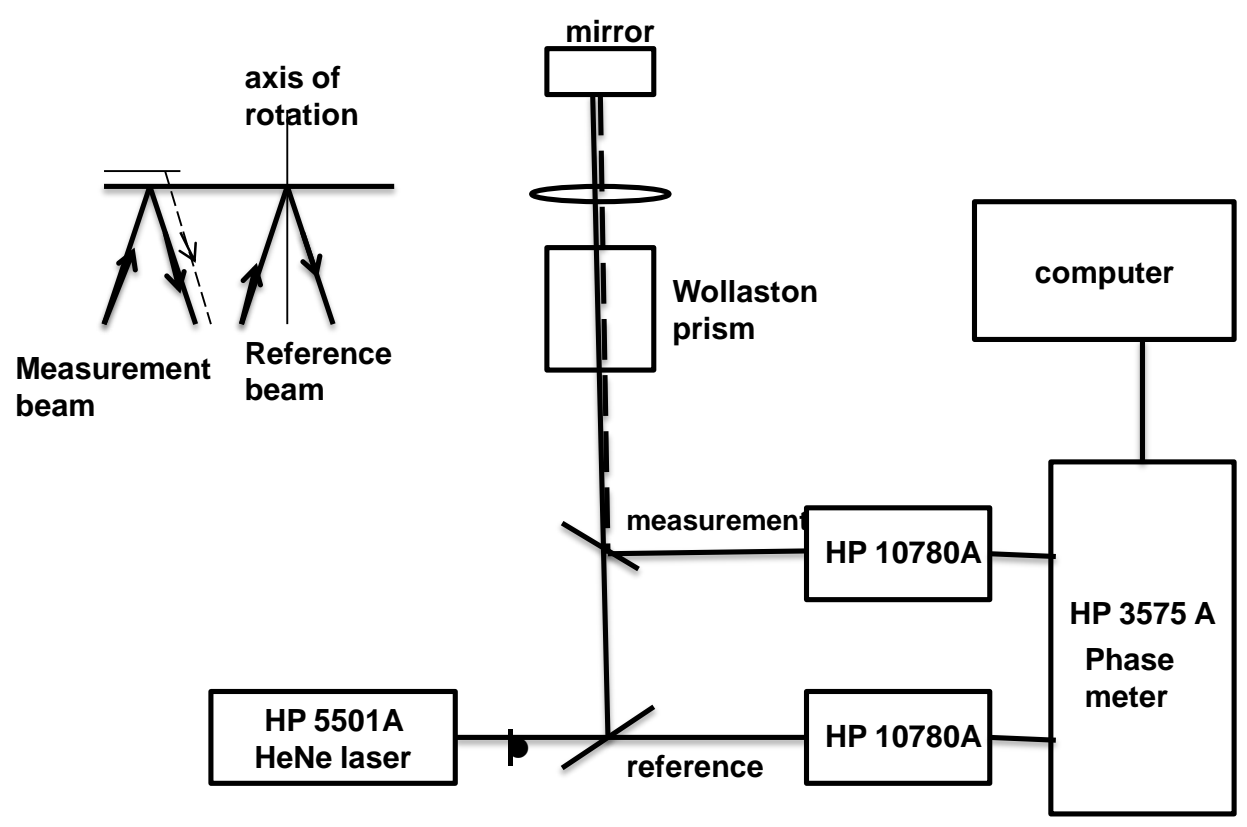

\section{Measurement of Temperature Changes}

\subsection{Application of Optical Heterodyne Detection Technique to Laser Cooling/Heating of Solids}

For the measurement of temperature changes in laser heating or cooling experiments, the heterodyne technique works equally well. However, the mathematical formulation is entirely different which is described below. Whenever a material is heated or cooled its length as well as refractive index change. A measurement of optical path length variation of a beam, which passes through the material, reveals the sample temperature change. So, prior knowledge of the temperature dependence of the material's refractive index and thermal expansion coefficient is needed. For the implementation of optical heterodyne technique a Mach-Zehnder interferometer configuration (Figure 2) is a straight forward approach whose theory is discussed below. The two orthogonally polarized beams of the laser are spatially separated by a PBS of which one is a reference beam $\left(v_{1}\right)$ and the other is the measurement beam $\left(v_{2}\right)$ that passes through the material under investigation. In this case, the material is heated or cooled by another laser beam that gets reflected by the dichroic mirror (DM), which is collinear with the probing beam but passes in the opposite direction (Figure 2). The laser beam either heats or cools the laser material depending on the sample and beam wavelength. Say $v_{1}$ and $v_{2}$ travel roughly equal distances, and their path length difference is within the coherence length of the laser. 
The refractive index of air and the material are represented by $\eta_{1}$ and $\eta_{2}$, respectively. $L_{1}$ and $L_{2}$ are the lengths of the interferometer arms and $\mathrm{L}_{3}$ is the sample length. $\mathrm{D}_{1}$ and $\mathrm{D}_{2}$ represent the optical path lengths of the reference and measurement beams which are given by

$$
\begin{gathered}
\mathrm{D}_{1}=\eta 1\left(\mathrm{~L}_{1}+\mathrm{L}_{2}\right) \\
\mathrm{D}_{2}=\eta_{1} \mathrm{~L}_{2}+\eta_{1}\left(\mathrm{~L}_{1}-\mathrm{L}_{3}\right)+\eta_{2} \mathrm{~L}_{3}
\end{gathered}
$$

Optical path length difference (OPD) is given by

$$
\mathrm{OPD}=\mathrm{D}_{2}-\mathrm{D}_{1}=\eta_{2} \mathrm{~L}_{3}-\eta_{1} \mathrm{~L}_{3}
$$

If we assume that the refractive index of air, $\eta_{1}=1$ then

$$
\mathrm{OPD}=\eta_{2} \mathrm{~L}_{3}-\mathrm{L}_{3}
$$

Here after, we drop the subscripts and use $\eta$ for the refractive index of the material and $L$ for sample length

$$
\mathrm{OPD}=\eta \mathrm{L}-\mathrm{L}
$$

As the sample temperature changes, $\eta$ and L change accordingly. Therefore, change in optical path length difference is given by

$$
\Delta(\text { opd })=\Delta(\eta \mathrm{L})-\Delta \mathrm{L}=\Delta \eta \mathrm{L}+\eta \Delta \mathrm{L}-\Delta \mathrm{L}
$$

Refractive index and sample length are temperature dependent. Therefore

$$
\begin{aligned}
& \Delta(o p d)=\frac{\partial \eta}{\partial T} \Delta T L+\eta \frac{\partial L}{\partial T} \Delta T-\frac{\partial L}{\partial T} \Delta T \\
& \Delta(o p d)=\eta L\left(\frac{1}{\eta} \frac{\partial \eta}{\partial T}+\frac{1}{L} \frac{\partial L}{\partial T}-\frac{1}{\eta L} \frac{\partial L}{\partial T}\right) \Delta T
\end{aligned}
$$

where

$$
\alpha=\frac{1}{L} \frac{\partial L}{\partial T}
$$

and

$$
\gamma=\alpha+\frac{1}{\eta} \frac{\partial \eta}{\partial T}
$$

The change in $O P D$ is simplified as

$$
\Delta(o p d)=\eta L\left(\gamma-\frac{\alpha}{\eta}\right) \Delta T
$$

Optical path length difference is related to the phase difference between the two beams as

$$
\Delta \Theta=\frac{2 \pi}{\lambda} \Delta(o p d)=\frac{2 \pi}{\lambda} \eta L\left(\gamma-\frac{\alpha}{\eta}\right) \Delta T
$$

By rearranging the above equation, temperature difference is estimated as

$$
\Delta T=\frac{\lambda \Delta \Theta}{2 \pi \eta L\left(\gamma-\frac{\alpha}{\eta}\right)}
$$

Probe laser wavelength is $632.8 \mathrm{~nm}$. The values of $\alpha$ and $\gamma$ are estimated from the sample data [23] using Equations (14) and (15). $\Delta \Theta$ can be measured to an accuracy of $0.1^{\circ}$ (resolution) with a phase 
meter (Model HP 3575A). For YAG material $\alpha=7.8 \times 10^{-6}, \gamma=11 \times 10^{-6}, \mathrm{~L}=0.0105 \mathrm{~m}$ and $\eta=1.821$. If $\Delta \Theta$ is $0.1^{\circ}$, then Equation (18) predicts a temperature change of $1.4 \mathrm{mK}$ (theoretical limit). This accuracy is comparable to that obtained with differential luminescence measurement [17]. However, the measurement of such a small change in temperature may not be possible because the accuracy is limited by the uncertainty, which is calculated by the propagation of errors [24], as

$$
\Delta T_{u}=\sqrt{\left(\frac{\partial T}{\partial \theta}\right)^{2}\left(\Delta \theta_{u}\right)^{2}+\left(\frac{\partial T}{\partial \lambda}\right)^{2}\left(\Delta \lambda_{u}\right)^{2}+\left(\frac{\partial T}{\partial L}\right)^{2}\left(\Delta L_{u}\right)^{2}+\left(\frac{\partial T}{\partial \eta}\right)^{2}\left(\Delta \eta_{u}\right)^{2}+\left(\frac{\partial T}{\partial \alpha}\right)^{2}\left(\Delta \alpha_{u}\right)^{2}+\left(\frac{\partial T}{\partial \gamma}\right)^{2}\left(\Delta \gamma_{u}\right)^{2}}
$$

where the subscript $u$ refers to uncertainty in the parameter and the partial derivatives are evaluated from Equation (18). The uncertainty in wavelength, $\Delta \lambda_{u}$ is zero because the probe laser wavelength does not change. So, the accuracy in $\Delta \mathrm{T}$ estimation depends on the uncertainties in the estimates of $\eta$, $\alpha, \gamma$, and $\Delta \Theta$ measurement.

\subsection{Rare-Earth Luminescence Sensors for Temperature Measurement}

\subsubsection{Fluorescence Lifetime Sensors}

In solids, excited rare-earth ions relax radiatively and non-radiatively [25] and the latter exhibits temperature dependence. Under pulsed laser excitation, the emission intensity varies as $I(t)=I_{0} \exp$ $(-t / \tau)$ where $\tau$ is the excited state lifetime. So, the measured lifetime, $\tau$ of the excited level, $i$ is related to both the relaxation rates $[25]$ as

$$
\tau^{-1}(i)=\sum_{i j} \omega_{r}+\sum_{i j} \omega_{n r}(T)
$$

where $\Sigma \omega_{\mathrm{r}}$ and $\Sigma \omega_{\mathrm{nr}}$ are the total radiative and non-radiative relaxation rates, to all the lower levels, $\mathrm{j}$. $\tau$ decreases linearly with temperature, $\mathrm{T}$ if $\omega_{\mathrm{nr}} \gg \omega_{\mathrm{r}}$. In general, this situation applies to many of the rare-earth ion doped glasses, for which the phonon frequency is larger [26]. At high temperatures, non-radiative relaxation dominates, and the lifetime varies empirically as [27]

$$
\ln \tau=\ln \tau_{0}+(\text { cons } \tan t) \frac{1}{T}
$$

Equation (21) predicts that $\ln \tau$ increases linearly with $1 / T$ (or $\tau$ decreases with $\mathrm{T}$ ). Accordingly, the fluorescence lifetime decreases with an increase in temperature. The temperature range over which it exhibits linear variation is dependent on the material characteristics. We have investigated several materials and only four materials exhibited such linear variation in a limited temperature range. The lifetime of ${ }^{5} \mathrm{~F}_{5}$ level of $\mathrm{Ho}^{3+}$-doped $\mathrm{CaF}_{2}$ decreased linearly from $380 \mu \mathrm{s}$ at room temperature to $188 \mu \mathrm{s}$ at $1000{ }^{\circ} \mathrm{C}$ [28]. The ${ }^{1} \mathrm{D}_{2}$ level of $\mathrm{Pr}^{3+}$-doped YAG predominantly emits at $617 \mathrm{~nm}\left({ }^{1} \mathrm{D}_{2} \rightarrow{ }^{3} \mathrm{H}_{4}\right)$ and its lifetime decreased linearly with temperature, from $187 \mu \mathrm{s}$ at $20{ }^{\circ} \mathrm{C}$ to $110 \mu \mathrm{s}$ at $1000{ }^{\circ} \mathrm{C}$ [28]. On exposure to a $308 \mathrm{~nm}$ laser beam, Samarium doped $\mathrm{CaF}_{2}$ revealed emission at 424, 560, 600, 620, and $690 \mathrm{~nm}$ and the sample color changed to bluish green due to the reduction of $\mathrm{Sm}^{3+}$ to $\mathrm{Sm}^{2+}$. The intensity of $424 \mathrm{~nm}$ peak decreased drastically with an increase in temperature [29], and the upper level lifetime decreased from $380 \mu$ s at $20{ }^{\circ} \mathrm{C}$ to $34 \mu \mathrm{s}$ at $85{ }^{\circ} \mathrm{C}$ (Figure 9). The slope of the plot is $5.9 \pm 0.4 \mu \mathrm{s} /{ }^{\circ} \mathrm{C}$. In other words, for each degree rise in temperature the lifetime decreased by $5.9 \mu \mathrm{s}$. This implies that the temperature can be measured to high accuracy. However, the accuracy is limited 
by the uncertainty whenever the data points scatter (Figure 9). We will use this illustration to estimate the uncertainty. In general, a plot of lifetime versus temperature varies linearly in a limited range, which is represented by

$$
\tau=\mathrm{mT}+\mathrm{b}
$$

where $\mathrm{b}$ and $\mathrm{m}$ represent intercept and slope respectively $(\mathrm{b}=518.8 \pm 22.7 \mu$ s and $\mathrm{m}=5.9 \pm 0.4$ $\mu \mathrm{s} /{ }^{\circ} \mathrm{C}$ ). If the lifetime measured at some unknown temperature is $\tau=200 \mu \mathrm{s}$, the corresponding sample temperature is estimated from the calibration plot as $54.5^{\circ} \mathrm{C}$. Equation (22) can be rearranged as

$$
\mathrm{T}=(\tau-\mathrm{b}) / \mathrm{m}
$$

Here, the accuracy in temperature estimation depends on the accuracies in $\tau, \mathrm{b}$ and $\mathrm{m}$ values. By the method of propagation of errors, the uncertainty in the temperature estimation is given by

$$
\delta T=\sqrt{\left(\frac{\partial T}{\partial \tau} \delta \tau\right)^{2}+\left(\frac{\partial T}{\partial b} \delta b\right)^{2}+\left(\frac{\partial T}{\partial m} \delta m\right)^{2}}
$$

where $\delta \tau, \delta b, \delta \mathrm{m}$, and $\delta \mathrm{T}$ are uncertainties in lifetime, intercept, slope and temperature, respectively, and are expressed by

$$
\frac{\partial T}{\partial \tau}=\frac{1}{m}, \frac{\partial T}{\partial b}=\frac{-1}{m} \text { and } \frac{\partial T}{\partial m}=\frac{b-\tau}{m^{2}}
$$

For the data shown in Figure 9, the uncertainties $\delta \tau=4$ (by assuming a $2 \%$ error in lifetime measurement), $\delta \mathrm{b}=22.7, \delta \mathrm{m}=0.4$, and the uncertainty in temperature measurement, $\delta \mathrm{T}=5.3$ (or 9.7\%). Fluorescence lifetime sensing of temperature was investigated by several other groups using rare-earth ion doped fibers, waveguides [30-32], crystals, glasses and powders [3].

Figure 9. Variation of $424 \mathrm{~nm}$ emission lifetime in $\mathrm{Sm}^{3+}$-doped $\mathrm{CaF}_{2}$ as a function of temperature [29].

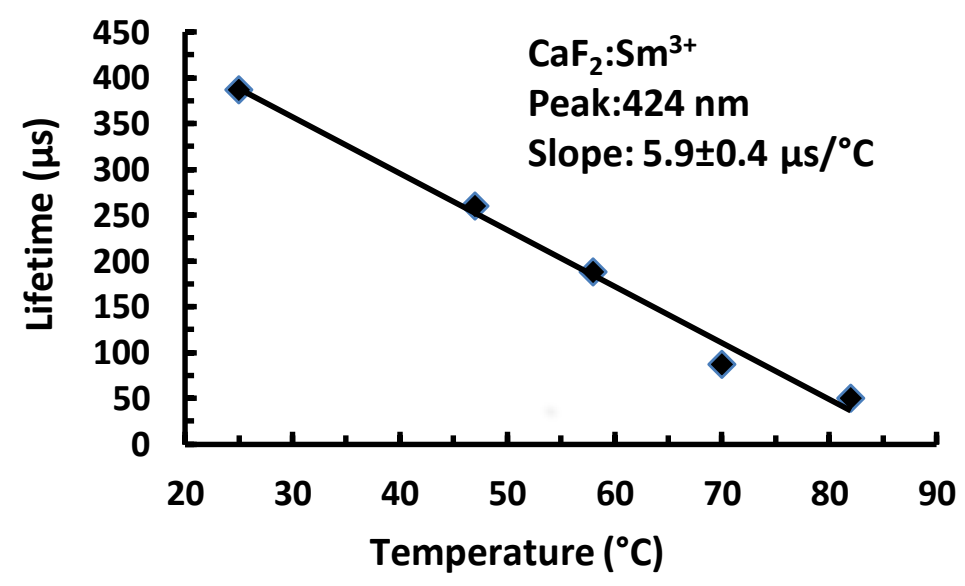




\subsubsection{Fluorescence Ratio Method}

Whenever ${ }^{4} \mathrm{~F}_{7 / 2}$ level of $\mathrm{Er}^{3+}$ is excited, it relaxes non-radiatively in cascade to the ${ }^{4} \mathrm{~S}_{3 / 2}$ level (Figure 10) and fluorescence occurs from the latter at $550 \mathrm{~nm}\left({ }^{4} \mathrm{~S}_{3 / 2} \rightarrow{ }^{4} \mathrm{I}_{15 / 2}\right)$. At higher sample temperatures, population is sustained in the higher multiplet, ${ }^{2} \mathrm{H}_{11 / 2}$, due to thermalization; and emission also occurs from ${ }^{2} \mathrm{H}_{11 / 2}$ at $530 \mathrm{~nm}\left({ }^{2} \mathrm{H}_{11 / 2} \rightarrow{ }^{4} \mathrm{I}_{15 / 2}\right)$. Accordingly, the measured lifetimes are same for both the emissions. The fluorescence intensity ratio of $530 \mathrm{~nm} / 550 \mathrm{~nm}$ bands increases with an increase in temperature. This phenomenon was also used in temperature sensing. The ratio of the emission intensities at temperature, $\mathrm{T}$ is given by [33]

$$
R=\frac{I_{20}}{I_{10}}=\frac{N_{2} v_{20} A_{20}}{N_{1} v_{10} A_{10}}=\frac{A_{20} g_{2} v_{20}}{A_{10} g_{1} v_{10}} e^{-\frac{\Delta E_{21}}{k T}}=(\text { constnt }) e^{-\frac{\Delta E_{21}}{k T}}
$$

where $\mathrm{g}_{\mathrm{i}}$ is the degeneracy of level $\mathrm{i}(\mathrm{i}=0,1,2)$, and $\mathrm{N}_{\mathrm{i}}$ is the population of levels ${ }^{2} \mathrm{H}_{11 / 2}$ (level 2) and ${ }^{4} \mathrm{~S}_{3 / 2}$ (level 1), $\Delta \mathrm{E}$ is the energy gap between levels 2 and 1 , and $\mathrm{kT}$ is the thermal energy. The emission intensity of a transition depends on the population, $\mathrm{N}_{\mathrm{i}}$ of the excited level, frequency $v_{\mathrm{i} 0}$ and the transition probability, $\mathrm{A}_{\mathrm{i} 0}$ to the ground level, 0 . The above equation reduces to

$$
\ln R=a-\frac{\Delta E_{21}}{k T}=a-\frac{b}{T}
$$

A plot of $\ln R$ versus $1 / \mathrm{T}$ decreases linearly with temperature, whereas $\ln R$ versus $\mathrm{T}$ increases linearly with $\mathrm{T}$. Such a linear variation was observed in a limited temperature range, up to $140{ }^{\circ} \mathrm{C}$ in $\mathrm{Er}^{3+}$-doped silica waveguide [31] and up to $96^{\circ} \mathrm{C}$ in $\mathrm{Er}^{3+}$-doped silica fiber [34].

Figure 10. Partial energy level diagram of $\mathrm{Er}^{3+}$-doped $\mathrm{LaF}_{3}$ depicting the excitation and emission wavelengths [22].

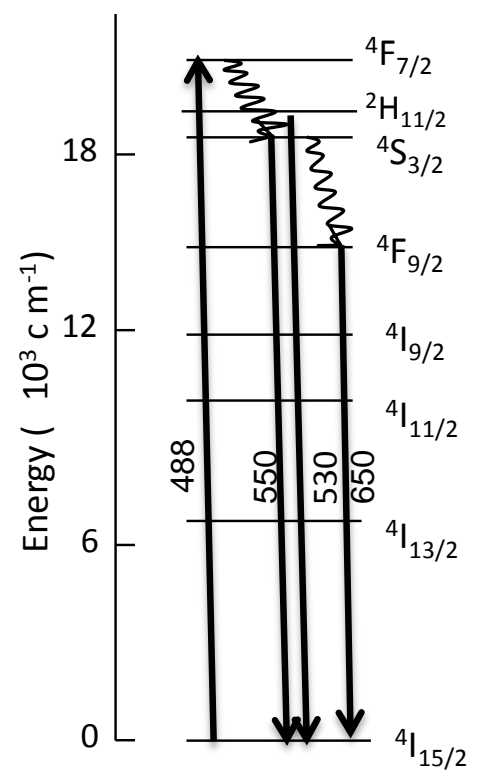

\subsection{Application of Michelson and Mach-Zehnder Interferometers to Measure Temperature Changes}

Interferometry deals with either fringe counting or phase measurement [5,7]. Fringes are produced by the superposition of two coherent beams of which one is a reference beam and the other is the 
measurement beam. The latter either passes through or gets reflected from the sample and its optical path length changes, whenever the sample is heated or cooled. So, the fringe intensity, measured by a detector, changes continuously with time, because of a change in the optical path length, $\eta \mathrm{L}$, where the sample length, $L$ and refractive index, $\eta$ are temperature dependent. Depending on the experimental configuration, a Michelson (Figure 11) [24] or Mach-Zehnder (Figure 12) [35] interferometer configuration is used. For a Michelson interferometer configuration, the optical path length difference (OPD) between the two beams is given by [24]

$$
\mathrm{OPD}=2 \mathrm{~L}(\eta-1)
$$

where $\mathrm{L}$ is the sample length and $\eta$ is its refractive index. For a temperature change $\Delta \mathrm{T}$, if the detector counts $\mathrm{N}$ fringes then the change in optical path length difference equals $\lambda \mathrm{N}$, where $\lambda$ is the laser wavelength. Temperature change is given by [24]

$$
\Delta T=\frac{\lambda N}{2 L \eta\left(\gamma-\frac{\alpha}{\eta}\right)}
$$

where the symbols have their usual meaning. The interferometric fringes produced by a Mach-Zehnder interferometer are shown in Figure 13. The detector output variation is shown in Figure 14. For a Mach-Zehnder interferometer, the temperature change is given by [35]

$$
\Delta T=\frac{\lambda N}{L \eta\left(\gamma-\frac{\alpha}{\eta}\right)}
$$

Here, the measurement accuracy is limited by the accuracies with which we can measure the fringe shift, index of the material $\eta$, coefficient of thermal expansion $\alpha$ and $\gamma$ of the material. Uncertainty in $\Delta \mathrm{T}$ is estimated by the propagation of errors. For a Mach-Zehnder interferometer, the temperature change is twice to that given by Equation (29) because of single pass configuration of the beam through the material. Michelson interferometer configuration was used to measure temperature changes produced by laser absorption in a $\operatorname{Pr}^{3+}$-doped YAG. A $488 \mathrm{~nm}$ laser was used to excite $\operatorname{Pr}^{3+}$ ions to ${ }^{3} \mathrm{P}_{0}$ level.

Figure 11. $\mathrm{Ar}^{+}$laser heats the sample. A HeNe laser was used to setup a Michelson interferometer configuration to measure laser heating of $\operatorname{Pr}^{3+}$-doped YAG crystal [24].

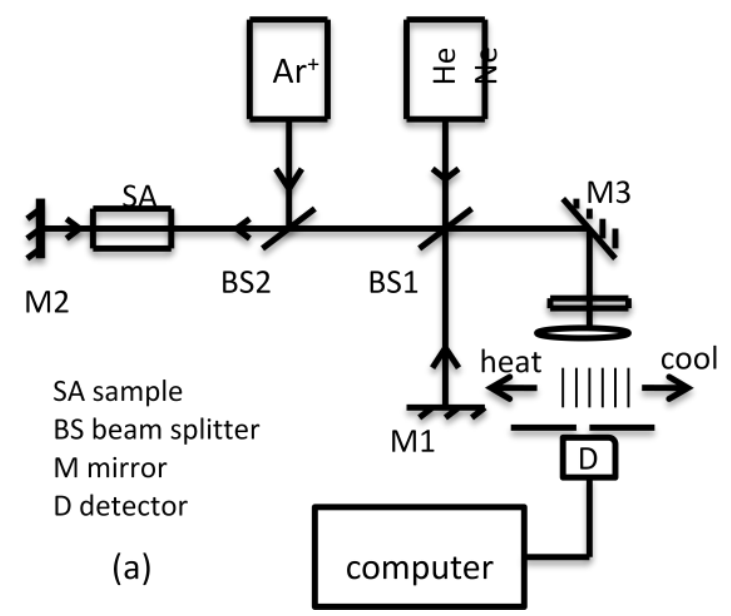


Figure 12. A $915 \mathrm{~nm}$ laser beam heats the YAG: $\mathrm{Yb}^{3+}$ sample. A $514.5 \mathrm{~nm}$ beam was used to setup a Mach-Zehnder interferometer to measure temperature changes [35].

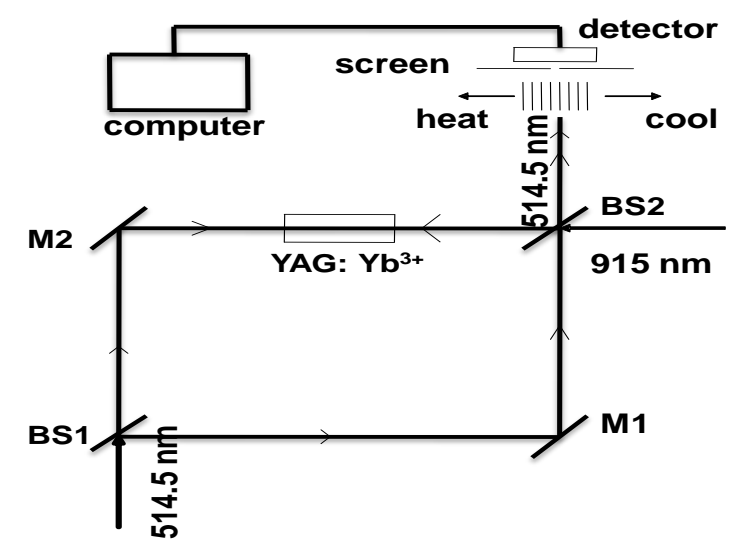

Figure 13. Interferometer fringes produced by a $514.5 \mathrm{~nm}$ laser. The dark spot is the pin hole in front of the detector [36].

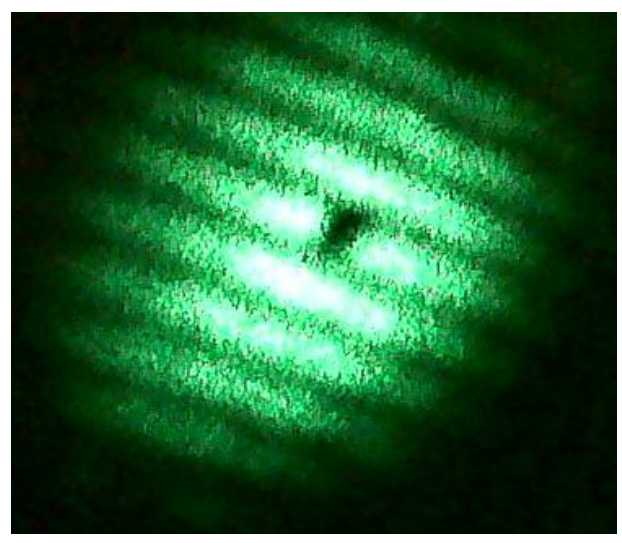

Figure 14. Detector output variation during heating, 0-1000 s (Ti: Sapphire laser on) and cooling, 1000-2000 s (Ti: Sapphire laser off) [35].

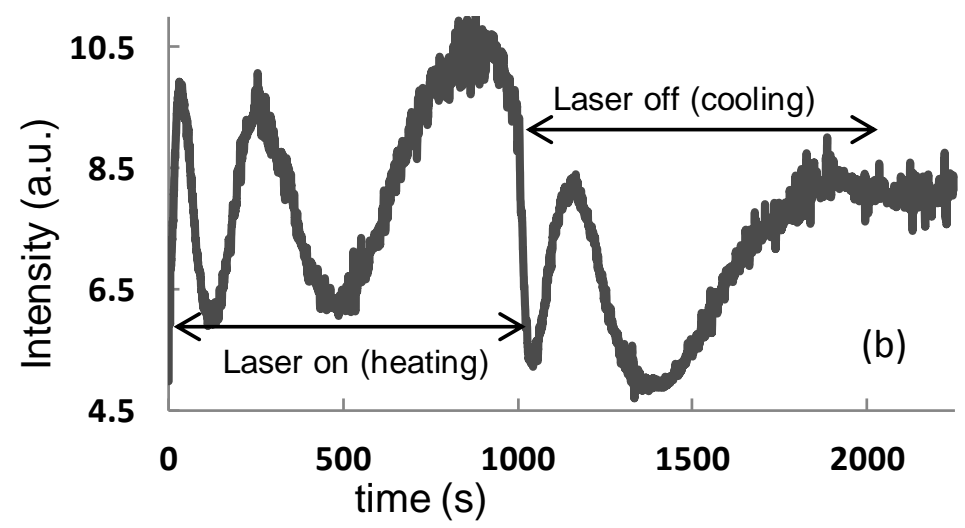

Non-radiative relaxation to ${ }^{1} \mathrm{D}_{2}$ causes laser heating. The change in temperature was probed by a $632.8 \mathrm{~nm}$ HeNe laser [24]. A Mach-Zehnder interferometer configuration was used to measure temperature changes in $\mathrm{Yb}^{3+}$-doped YAG caused by laser absorption at $915 \mathrm{~nm}$. A $915 \mathrm{~nm}$ laser 
excites $\mathrm{Yb}^{3+}$ ions from the ground state to the excited state, ${ }^{2} \mathrm{~F}_{5 / 2}$. Excited ions undergo non-radiative relaxation processes which heats up the material, along the beam path. The change in temperature was measured with $514.5 \mathrm{~nm}$ laser beam by setting up a Mach-Zehnder interferometer [35] and the fringes are shown in Figure 13. The intensity of the fringe pattern varied continuously with time, and the detector output followed such a variation (Figure 14). The crystal was heated by the Ti: Sapphire laser beam from 0-1000 s and the material cooled down from 1000-2000 s, when the Ti: Sapphire laser beam was blocked. As soon as the Ti: Sapphire laser was on, the non-radiative relaxation starts, and the material is heated. So the fringe period is small in the beginning. Eventually, the material temperature approaches the equilibrium state, causing the fringe period to increase. Similar situation occurs when the heating laser beam was blocked, from 1000-2000 s. For a $300 \mathrm{~mW}$ of $915 \mathrm{~nm}$ laser beam, the sample temperature increased by $6.9{ }^{\circ} \mathrm{C}$ [35]. This is a unique technique, which measures heating or cooling along the beam path, in real time. The accuracy with which $\Delta \mathrm{T}$ can be estimated depends on the accuracy of the other parameters $\alpha, \beta$, and $\gamma$.

\section{Application of Mach-Zehnder Interferometer Technique for the Measurement of Changes in Solution Concentration or Refractive Index in a Crystal Growth Experiment}

A typical solution growth experiment is shown in Figure 15. Crystals are grown by mounting a seed crystal on a cold finger whose temperature is slightly less than that of the solution [37]. During growth, solute particles migrate from solution to the seed crystal. As a result, the refractive index of the solution changes in the vicinity of the seed crystal. This change can be measured by setting up a Mach-Zehnder interferometer, whose measurement beam passes in front of the seed crystal in the solution, as shown in Figure 15. The optical path lengths of the reference and the measurement beams are given by

$$
\mathrm{L}_{1}=\eta_{1}\left(\mathrm{l}_{1}+\mathrm{l}_{2}\right)
$$

and

$$
\mathrm{L}_{2}=\eta_{1}\left(\mathrm{l}_{2}+\mathrm{a}_{1}+\mathrm{a}_{2}\right)+\eta_{2}\left(\mathrm{~b}_{1}+\mathrm{b}_{2}\right)+\mathrm{s} \eta_{3}
$$

where $\eta_{i}(i=1,2$ and 3$)$ are refractive indices of air, glass and solution, $l_{1}$ and $l_{2}$ are the interferometer arm lengths in air. The measurement beam passes through air, glass windows and solution whose path lengths are $\left(l_{2}+a_{1}+a_{2}\right),\left(b_{1}+b_{2}\right)$ and $S$, respectively.

The phase difference $\Theta_{d}$ between the two beams of the interferometer is given by

$$
\Theta_{d}=\frac{2 \pi}{\lambda}\left[\eta_{1}\left(a_{1}+a_{2}-l_{1}\right)+\eta_{2}\left(b_{1}+b_{2}\right)+s \eta_{3}\right]
$$

As the material grows solute particles migrate from solution along the beam path towards the crystal surface, thus changing the refractive index of the solution. Hence, the third term in the bracket changes during crystal growth while the first two terms remain constant. The magnitude of variation in the phase difference is given by

$$
\Delta \Theta_{d}=\frac{2 \pi}{\lambda} s \Delta \eta_{3}
$$


$\Delta \Theta_{\mathrm{d}}$ can be measured experimentally. During crystal growth the fringes appear to be moving to the naked eye. If the fringe intensity is measured by a detector its output exhibits periodic variation (Figure 16). So the change in index for a given change in phase difference is given by

$$
\Delta \eta_{3}=\frac{\lambda}{2 \pi s} \Delta \Theta_{d}=\frac{\lambda}{s}
$$

where $\Delta \Theta_{\mathrm{d}}=2 \pi$ is the phase difference between two successive peaks (Figure 17). Refractive index changes with a change in the concentration of solute particles, $\mathrm{c}$ as

$$
\Delta \eta_{3}=\frac{\partial \eta}{\partial c} \Delta c
$$

Refractive index variation $\frac{\partial \eta}{\partial c}$ can be measured experimentally. For a $2 \pi$ phase change $\left(\Delta \Theta_{d}=2 \pi\right)$, the concentration change can be estimated as

$$
\Delta c=\frac{\lambda}{\left(\frac{\partial \eta}{\partial c}\right) s}
$$

The change $\Delta \eta_{3}$ can be correlated to growth rate if we measure the growth simultaneously by an alternate technique, such as heterodyne detection technique [20] or from laser reflectance measurements [37]. In Equation (37), the laser wavelength $(\lambda)$ and the solution path length (s) do not change. So, the uncertainty in the estimation of concentration change depends on the accuracy with which $\frac{\partial \eta}{\partial c}$ was measured. According to Equation (35) $\Delta \eta_{3}$ equals $\lambda / s$ if $\Delta \Theta_{d}=2 \pi$. The optical mounts/beams are subjected to random vibrations. So the fringe intensity and hence the electrical output of the detector fluctuates (Figure 16). That is why the fringes shown in Figure 16 are not uniform in shape. This will result in a maximum error of $10 \%$ in the estimation of $\Delta \eta_{3}$. So the maximum error in the estimation of change in concentration is likewise $10 \%$.

Figure 15. Optical configuration of the Mach-Zehnder interferometer configuration for the measurement of changes in solution concentration/index of refraction. Measurement beam passes in front of the seed crystal inside the solution. Laser 2 is used for growth rate measurement [37].

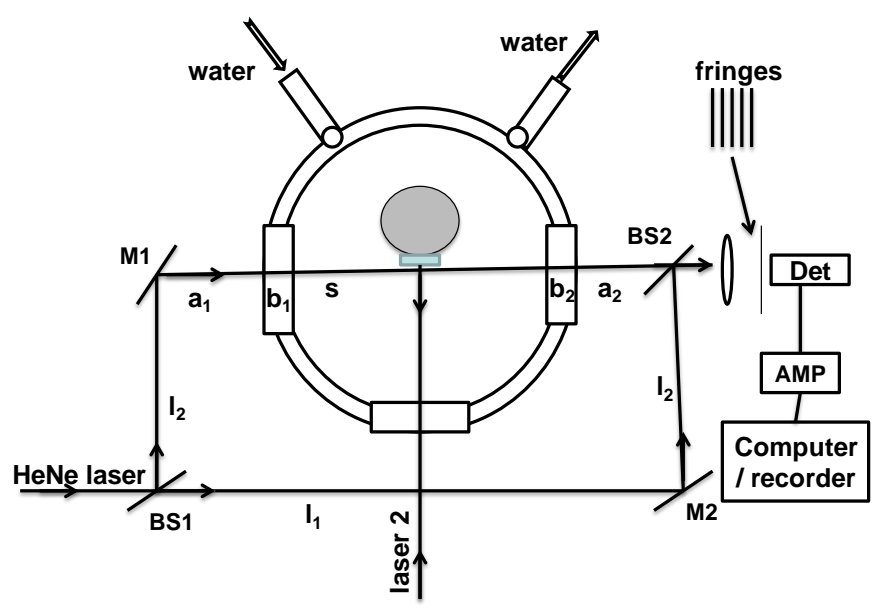


Figure 16. Temporal dependence of the detector output (of Figure 15) reveals fringe behavior [37].

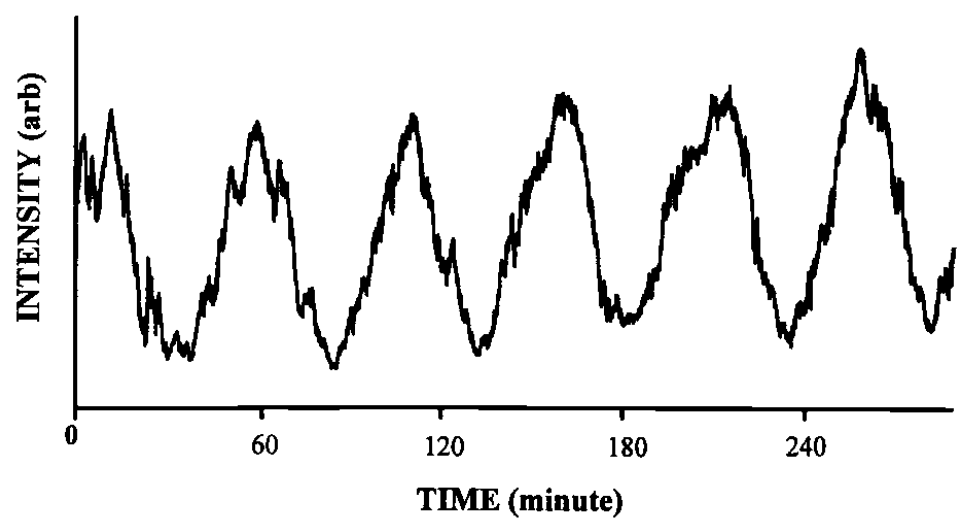

Figure 17. The data shown in Figure 16 are plotted to reveal phase variation as a function of time [37].

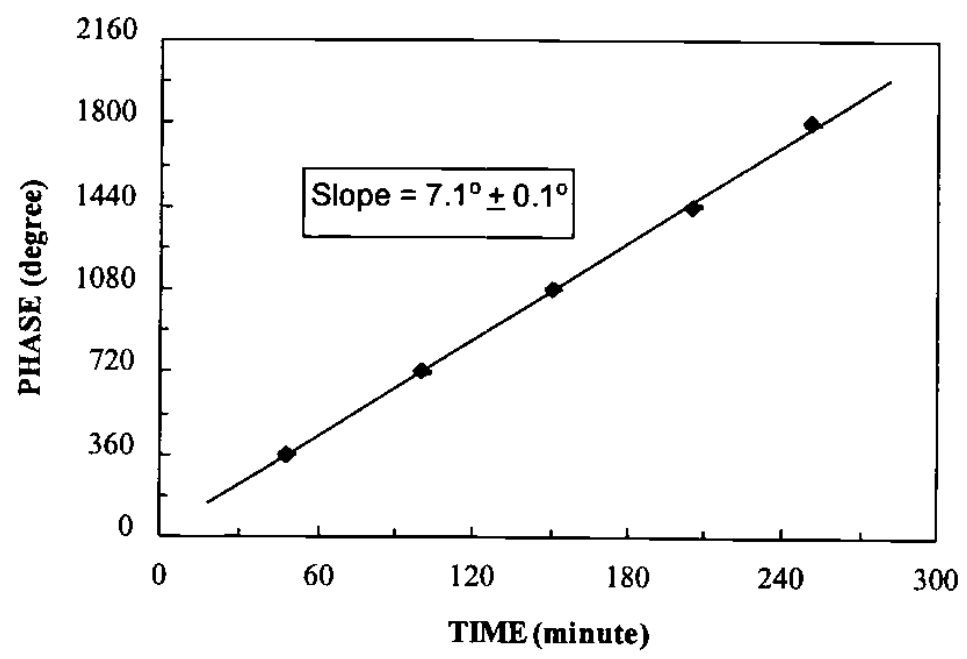

\section{Conclusions}

Optical heterodyne detection theory has been developed for the measurements of crystal growth, surface roughness of mirrors and laser heating/cooling of solids. The technique was demonstrated for in situ measurement of crystal growth and surface roughness of coated optics. Michelson and Mach-Zehnder interferometers were applied for the measurement of temperature changes in laser heating of $\mathrm{Pr}^{3+}$-doped YAG and $\mathrm{Yb}^{3+}$-doped YAG. We have demonstrated the suitability of rare-earth ion doped crystals and glasses for temperature measurement using fluorescence ratio method, and lifetime sensing. Mach-Zehnder interferometer was also demonstrated for the measurement of solution concentration changes. Rare-earth luminescence sensors and interferometers are suitable for temperature measurements in a variety of situations including jet plumes, combustion studies, corrosive atmospheres and many others. Interferometer arrangement is relatively difficult to set up, and require accurate values for the coefficients of thermal expansion and refractive index. Techniques that involve interferometers are suitable for certain unique situations and provide absolute values of temperature changes. 
In Michelson and Mach-Zehnder interferometer techniques, the reference and measurement beams travel along different paths and so these techniques are sensitive to vibrations. For in-situ measurements, Michelson, M-Z, optical and heterodyne techniques are desirable. Of these, the last one is the most sensitive technique. For assessment or surface topography, phase shifting interferometry and dynamic interferometry techniques are suitable, which have sub nanometer sensitivity and are suitable for very fast assessment of large surfaces. These techniques have sub nanometer sensitivity in depth profile measurement and micron or better in spatial resolution. For laser heating or cooling studies of solids optical heterodyne, Michelson and Mach-Zehnder interferometer are suitable. Of these, only optical heterodyne technique can reveal temperature changes of $\mathrm{mK}$, in real time. Dynamic interferometry technique is not suitable for real time measurements of temperature changes induced by a laser beam along the beam path. When the temperature or length changes are large and if one is interested in average changes over a long period of time then Michelson, Mach-Zehnder and heterodyne techniques are equally good. When the changes in temperature are small, optical heterodyne technique is superior to other techniques. If one is interested in absolute temperature measurement then rare-earth luminescence or spectral measurements are better [18,22]. For the measurement of flatness of optics, inexpensive techniques like Fizeau, Twyman-Green or shear interferometers will do just fine. For surface roughness measurements, dynamic interferometry and other phase shifting interferometry techniques are superior and faster. Optical heterodyne technique is suitable for small surface areas whereas dynamic interferometry is suitable for large surface areas. For accurate measurement of laser wavelength, Michelson type interferometers are suitable. If one is interested in average growth rate over a period of several minutes, even a simple reflection measurement (front and back surface reflections) reveals reasonable estimate, provided the sample thickness is smaller. Optical heterodyne technique requires a stable two frequency laser and hence it is relatively expensive when compared to reflection or conventional Michelson type interferometers.

\section{Conflicts of Interest}

The author declares no conflict of interest.

\section{References}

1. Hariharan, P. Optical Inteferometry; Elsevier/Academic Press: New York, NY, USA, 2003.

2. Zhou, J.J.; Li, Y.; Thompson, P.; Chu, R.; Lee, H.P. Continuous in situ growth rate extraction using pyrometric interferometry and laser reflectance measurement during molecular beam epitaxy. J. Electron. Mater. 1997, 26, 1083-1089.

3. Allison, S.W.; Gillies, G.T. Remote thermometry with thermographic phosphors: Instrumentation and applications. Rev. Sci. Instrum. 1997, 68, 2615-2650.

4. Koechner, W.; Bass, M. Solid State Lasers; Springer-Verlag: New York, NY, USA, 2003.

5. Gasvik, K.J. Optical Metrology; John Wiley \& Sons: New York, NY, USA, 1995.

6. Hilfiker, J. Emerging applications of spectroscopic ellipsometry. Glass Coatings 2007, 1, 24-27.

7. Hariharan, P.; Oreb, B.F.; Eiju, T. Digital phase-shifting interferometry: A simple error-compensating phase calculation algorithm. Appl. Opt. 1987, 26, 2504-2506. 
8. Kanzaki, N.; Onuma, K.; Ito, A.; Teraoka, K.; Tateishi, T; Tsutsumi, S. Direct growth rate measurement of Hydroxyapatite single crystal by Moiré phase shift interferometry. J. Phys. Chem. 1998, 102, 6471-6476.

9. Hariharan, P. Optical Holography: Principles, Techniques, and Applications; Cambridge University Press: Cambridge, UK, 2004.

10. Gao, F.; Muhamedsalih, H.; Jiang, X. Surface and thickness measurement of a transparent film using wavelength scanning interferometry. Opt. Express 2012, 20, 21450-21456.

11. Zuiker, C.D.; Gruen, D.M.; Krauss, A.R. In situ laser reflectance interferometry measurement of diamond film growth. J. Appl. Phys. 1996, 79, 3541-3546.

12. Yoshizaki, I.; Tsukamoto, K.; Yamazaki, T.; Murayama, K.; Oshi, K.; Fukuyama, S.; Shimaoka, T.; Suzuki, Y.; Tachibana, M. Growth rate measurements of lysozyme crystals under microgravity conditions by laser interferometry. Rev. Sci. Instr. 2013, 84, doi:10.1063/1.4826090.

13. Masuda, K.; Erskine, D.; Anderson, O. Differential laser interferometer for thermal expansion measurements. Am. Mineral. 2000, 85, 279-282.

14. Farahani, M.A.; Torsten Gogolla, T. Spontaneous raman scattering in optical fibers with modulated probe light for distributed temperature raman remote sensing. J. Lightw. Technol. 1999, 17, 1379-1383.

15. Zhan, Y.; Wu, H.; Yang, Q.; Xiang, S.; He, H. Fiber Bragg grating sensors for high temperature measurement. Opt. Lasers Eng. 2008, 46, 349-354.

16. Liao, S.C.; Xu, Z.; Izatt, J.A.; Alcala, J.R. Real-time frequency domain temperature and $\mathrm{O}_{2}$ sensor with a single optical fiber. IEEE Trans. Biomed. Eng. 1997, 44, 1114-1121.

17. Patterson, W.M.; Seletskiy, D.V.; Sheik-Bahae, M.; Epstein, R.I.; Hehlen, M.P. Measurement of solid-state optical refrigeration by two-band differential luminescence thermometry. J. Opt. Soc. Am. B 2010, 27, 611-618.

18. Chubb, D.L.; Wolford, D.S. Rare-earth optical temperature sensor. Rev. Sci. Instrum. 2000, 71, 2233-2240.

19. Sommergren, G.E. Optical heterodyne profilometry. Appl. Opt. 1981, 20, 610-618.

20. Kim, Y.; Reddy, B.R.; George, T.G.; Lal, R.B. Optical heterodyne interferometry technique for solution crystal growth rate measurement. Opt. Eng. 1998, 37, 616-621.

21. George, M.C.; Reddy, B.R.; Jagannath, H.; Perera, G.M.; Venkateswarlu, P. Measurement of Surface Roughness Using a Noncontact Profilometer. In Laser Spectroscopy and Nonlinear Optics of Solids; Radhakrishna, S., Tan, B.C., Eds.; Narosa Publishing House: Delhi, India, 1991; pp. 147-152.

22. Kamma, I.; Kommidi, P.; Reddy, B.R. Design of a high temperature sensing system using luminescent lifetime measurement. Rev. Sci. Instrum. 2008, 79, doi:10.1063/1.2981704.

23. Fan, T.Y.; Daneu, J.L. Thermal coefficients of the optical path length and refractive index in YAG. Appl. Opt. 1998, 37, 1635-1637.

24. Farley, C.W.; Reddy, B.R. Interferometric measurement of laser heating in Praseodymium doped YAG crystal. Appl. Opt. 2011, 50, 526-531.

25. Weber, M.J. Probabilities for radiative and non-radiative decay of $\mathrm{Er}^{3+}$ in $\mathrm{LaF}_{3}$. Phys. Rev. 1967, $157,262-272$. 
26. Layne, C.B.; Lowdermilk, W.H.; Weber, M.J. Multiphonon relaxation of rare-earth ions in glasses. Phys. Rev. 1977, B16, 10-20.

27. Shepard, C.L.; Cannon, B.D.; Khaleel, M.A. Determination of temperature in glass with a fluorescence method. Int. J. Heat Mass Transfer 2001, 44, 4027-4034.

28. Kamma, I.; Kommidi, P.; Reddy, B.R. High temperature measurement using luminescence of $\mathrm{Pr}^{3+}$ doped YAG and $\mathrm{Ho}^{3+}$ doped $\mathrm{CaF}_{2}$. Phys. Stat. Sol. C 2009, 6, S187-S190.

29. Kommidi, P.; Reddy, B.R. Fluorescence lifetime sensing of temperature. Proc. SPIE 2006, 6377, doi:10.1117/12.695382.

30. Wade, S.A.; Collins, S.F.; Baxter, D.W. Fluorescence intensity ratio technique for optical fiber point temperature sensing. J. Appl. Phys. 2003, 94, 4743-4756.

31. Messias, D.N.; Vermelho, M.V.D.; Gouveia-Neto, A.S.; Aitchison, J.S. All optical integrated upconversion fluorescence based point temperature sensing system using $\mathrm{Er}^{3+}$ doped silica-on-silicon waveguides. Rev. Sci. Instrum. 2002, 73, 476-479.

32. Forsyth, D.I.; Sun, T.; Grattan, K.T.V.; Wade, S.A.; Collins, S.F. Characteristics of doped optical fiber for fluorescence-based fiber optic temperature systems. Rev. Sci. Instrum. 2003, 74, 5212-5218.

33. Shen, Y.; Wang, X.; He, H.; Lin, Y.; Nan, C. Temperature sensing with fluorescence intensity ratio technique in epoxy-based nanocomposite filled with $\mathrm{Er}^{3+}$-doped 7YSZ. Compos. Sci. Technol. 2012, 72, 1008-1011.

34. Paez, G.; Strojnik, M. Erbium-doped optical fiber fluorescence temperature sensor with enhanced sensitivity, a high signal-to-noise ratio and a power ratio in the 520-530- and 550-560-nm bands. Appl. Opt. 2003, 42, 3251-3258.

35. Reddy, B.R.; Kamma, I.; Kommidi, P. Optical sensing techniques for temperature measurement. Appl. Opt. 2013, 52, B33-B39.

36. Farley, C.W.; Reddy, B.R. Mach-Zehnder interferometric measurement of laser heating/cooling in $\mathrm{Yb}^{3+}$ : YAG. Proc. SPIE 2011, 7951, doi:10.1117/12.873944.

37. Kim, Y.K.; Reddy, B.R.; Lal, R.B. Laser and Mach-Zehnder interferometry for solution crystal growth rate and concentration variation measurement, in "Laser interferometry IX: Applications". Proc. SPIE 1998, 3479, 172-180.

(C) 2014 by the authors; licensee MDPI, Basel, Switzerland. This article is an open access article distributed under the terms and conditions of the Creative Commons Attribution license (http://creativecommons.org/licenses/by/3.0/). 